Solveig Wallberg Jonsson: None declared, Solbritt Rantapaa Dahlqvist: None declared, George Karpouzas Grant/research support from: Pfizer, Consultant for: Sanofi-Genzyme-Regeneron, Janssen, Roche-Genentech, Pfizer, Speakers bureau: BMS, Sanofi-Genzyme-Regeneron, Janssen, Roche-Genentech, Patrick Dessein: None declared, Linda Tsang: None declared, Hani El-Gabalawy: None declared, Carol Hitchon: None declared, Virginia Dr. Pascual: None declared, Irazu Contreras-Yáñez : None declared, Petros Sfikakis: None declared, Miguel A González-Gay Grant/research support from: Prof. MA Gonzalez-Gay received grants/ research supports from Abbvie, MSD, Jansen and Roche., Speakers bureau: Consultation fees/participation in company sponsored speaker's bureau from Pfizer, Lilly, Sobi, Celgene, Novartis, Roche and Sanofi., Cynthia S. Crowson: None declared, Anne Grete Semb: None declared DOI: 10.1136/annrheumdis-2019-eular.792

\section{SAT0110 HIGH SERUM LONG-CHAIN OMEGA-3 FATTY ACIDS ARE ASSOCIATED WITH 6-MONTHLOWER DISEASE ACTIVITY IN EARLY RA: RESULTS FROM THE ESPOIR COHORT}

Johanna Sigaux ${ }^{1}$, Camille de Brauer Buscail ${ }^{2}$, Chantal Julia², Rene Marc Flipo ${ }^{3}$, Alain Cantagrel ${ }^{4}$, Bernard Bannwarth ${ }^{5}$, Francois Laporte $^{6}$, Marie-

Christophe Boissier', Luca Semerano ${ }^{1}$. ${ }^{1}$ INSERM UMR 1125, Université Paris 13, Bobigny, France; ${ }^{2}$ EREN UMR U1153 Inserm/U1125 Inra/Cnam/Univ Paris 13, Bobigny, France; ${ }^{3}$ Department of Rheumatology, Lille University Hospital, 59037, Lille, France; ${ }^{4}$ Centre de Rhumatologie, CHU de Toulouse, 31059, Toulouse, France; ${ }^{5}$ Service de rhumatologie, hôpital Pellegrin, $\mathrm{CHU}$ de Bordeaux, Bordeaux, France; ${ }^{6}$ Département de Biochimie-Pharmacologie-Toxicologie CHU de Grenoble., Grenoble, France

Background: Dietary long-chain polyunsaturated fatty acids (PUFAs) of the $\mathrm{n} 3$ and $\mathrm{n} 6$ families are involved in immune homeostasis and can contribute to modulate inflammatory processes. Dietary intake of n3PUFA (by either supplementation or fish consumption) has been inversely associated with RA incidence in different populations. Some evidence suggests that, in subject at high risk of developing rheumatoid arthritis (RA), high erythrocyte membrane content in long-chain n3-PUFA is associated with lower ACPA prevalence and progression to RA. While long-chain n6PUFA are considered mainly proinflammatory, some, like gamma-linolenic acid (GLA), are endowed with anti-inflammatory activity, and the erythrocyte content in $n 6$ linoleic acid was inversely associated with the development of RA in a nested-case control study. No study hitherto evaluated the longitudinal association between individual PUFA profile and disease activity in early RA.

Objectives: To characterize serum profiles of PUFA and to determine their association with baseline variables and with 6-month disease activity in a cohort of patients with early RA.

Methods: Serum PUFAs were quantified by gas chromatography-mass spectrometry (GC/MS) in 594 patients with early RA at the time of recruitment in the French Etude et Suivi des POlyarthrites Indifférenciées Récentes (ESPOIR) cohort. Principal component analysis on 19 serum fatty acids with 14 to 22 carbon atoms allowed to determine 3 orthogonal patterns of baseline serum PUFA. Pattern 1 included high proportions of short-chain fatty acids, pattern 2 was high in $n 3$ long-chain PUFA (EPA et DHA), pattern 3 was rich in n6 long-chain fatty acids (among which, GLA had the highest concentration). Patients were stratified in tertiles according to how much the fitted into each pattern, with tertile 1 meaning lowest and tertile 3 highest fitting into the pattern. The association of PUFA patterns with baseline variables was tested in univariate analysis. The association with 6-month disease activity (with high disease activity defined as DAS28 $>5.1$ ) was tested in multivariable analysis after adjustment on baseline CRP, corticosteroid and/or NSAIDs use, socioeconomic status, ACPA and RF positivity, traditional and biologic DMARDs treatment between 0 and 6 months after inclusion.

Results: In baseline univariate analysis, pattern 1 was associated with high BMI, active smoking and with non-Caucasian origin. Profile 2 was associated with higher socioeconomic status and inversely associated with DAS28 and CRP. Profile 3 was associated with ACPA positivity and higher baseline CRP. In multivariate analysis, fitting into pattern 2 and 3 was associated with lower odds of having active disease after 6 months (OR for tertile 3 vs. tertile 1 : $0.49(95 \% \mathrm{Cl} 0.25$ to $0.97, \mathrm{p}<0.05)$ for pattern 2 and 0.51 (0.28 to 0.95 ) for pattern 3 , respectively).

Conclusion: In a cohort of early RA patients, we could identify a high serum long-chain n3PUFA profile associated with low disease activity between 0 and 6 months of follow-up in the cohort. This is consistent with a presumed immunomodulatory action of n3PUFA. Despite the baseline association with disease severity features, like ACPA positivity and high CRP, a high serum long-chain n6PUFA profile is also associated with 6-month lower disease activity, possibly due to the presence of high concentrations of GLA in this profile.

Disclosure of Interests: Johanna Sigaux Speakers bureau: celgene, Camille de Brauer Buscail: None declared, Chantal Julia: None declared, Rene Marc Flipo: None declared, Alain Cantagrel: None declared, Bernard Bannwarth: None declared, Francois Laporte: None declared, marie-Christophe Boissier Grant research support from: Pfizer MSD, Speakers bureau: Pfizer Lilly Biogen, Luca Semerano Grant/research support from: pfizer, Speakers bureau: pfizer, roche, msd.

DOI: 10.1136/annrheumdis-2019-eular.5911

\section{SAT0111 RED CELL DISTRIBUTION WIDTH IS A MARKER OF EARLY RESPONSE TO METHOTREXATE IN RHEUMATOID ARTHRITIS}

Daniele Soddu ${ }^{1},{ }^{1,2}$ Mattia Bellan $^{3}$, Erika Zecca ${ }^{1}$, Alessandro Croce ${ }^{1}$, Roberta Pedrazzoli ${ }^{1}$, Daniele Sola ${ }^{1},{ }^{1,2}$ Mario Pirisi ${ }^{3},{ }^{1,2}$ Pierpaolo Sainaghi ${ }^{3} .{ }^{1} A O U$ "Maggiore della Carità" Hospital, Internal Medicine Division, Immunorheumatology Unit, Novara, Italy; ${ }^{2}$ Università del Piemonte Orientale, Department of Translational Medicine, Novara, Italy, ${ }^{3}$ Interdisciplinary Center of Autoimmune DiseasesIRCAD, Novara, Italy

Background: Red blood cell distribution width (RDW) has recently emerged as a possible surrogate biomarker of inflammation. Its potential role as prognostic biomarker has not been evaluated yet in Rheumatoid Arthritis (RA).

Objectives: The following study aims to evaluate the potential prognostic role of RDW in the prediction of early response to methotrexate (MTX).

Methods: We performed a retrospective analysis of clinical records of patients affected by RA, according to ACR/Eular classification Criteria. The baseline RDW was recorded and correlated with disease activity scores and inflammatory markers. The response to treatment was evaluated at the 3-months follow-up visit according to Eular response criteria. Results: We selected 88 patients (58 females, 65.9\%), with a median age of 62 [52-69] years. 61 patients were positive for Rheumatoid Factor (RF) and/or anticitrullinated antibodies - anti-CCP (69.3\%). The median DAS28 for Erythrocyte sedimentation rate (DAS28ESR) at the diagnosis was 4.16 [3.33-5.00] while the median RDW was 14.0 [13.1-14.9]\%. The baseline RDW was directly associated to $\operatorname{CRP}(\rho=0.250 ; \mathrm{p}=0.02)$, but not with ESR $(p=0.32)$ or DAS28ESR $(p=0.40)$.

All the patients received methotrexate (MTX) at a median dose of 10 [10-15] $\mathrm{mg} /$ week, $79(89.8 \%)$ also received prednisone (median dose 5 [5-10] $\mathrm{mg} /$ day) and 20 hydroxychloroquine in addition to MTX. A significant trend towards a larger RDW was reported from patients with a good Eular response at three months (13.5 [13.0-14.4]\%) to those with a moderate response $(14.0[13.2-14.7] \%)$ and finally to those with a poor response (14.6 [13.6-16.0]\%; $\mathrm{p}=0.004)$. At logistic regression, RDW $(p=0.02)$, but not CRP or ESR $(p=0.70$ and $p=0.37$ respectively), was a predictor of a good Eular response.

Moreover, in a further logistic regression model, baseline RDW $(p=0.03)$ and baseline DAS28ESR $(p=0.0009)$ were the only predictors of a good Eular response at three months, while age, gender, MTX and prednisone dosage and seropositivity did not fit the model.

After the treatment RDW significantly increases (14.7 [14.0-15.7]\%) and the variation of RDW from baseline is inversely related to the variation of DAS28ESR ( $\rho=-0.263 ; p=0.02$ ). The increase of RDW is significantly higher in patients with a good Eular response, with a trend towards a smaller increase in case of moderate and poor response $(0.95$ [0.35$1.35] \%$ vs. $0.65[0.1-1.9] \%$ vs. $0.2[-0.62-0.77] \% ; p=0.015)$.

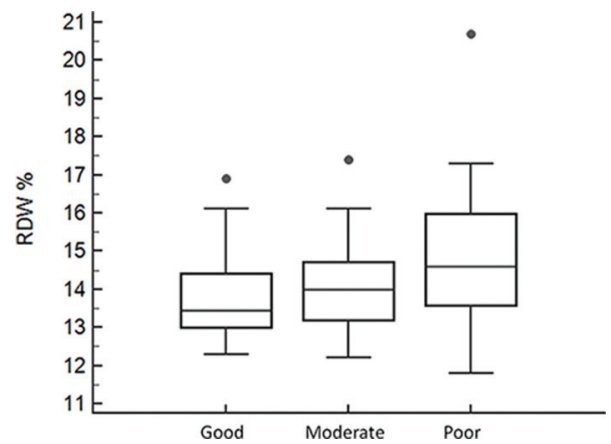

